\title{
Research on Parameters Tuning of PID Controller Based on Critical Proportioning Method
}

\author{
Xuewen Shi, Qinglan Wang ${ }^{*}$ and Yuqiang Wu \\ College of Engineering, Qufu Normal University, Rizhao 276826, China \\ ${ }^{*}$ Corresponding author
}

\begin{abstract}
Critical proportioning method is an important and widely used method for PID engineering tuning. Since textbooks only introduce PID parameters tuning steps and simulation examples, without giving the value of critical proportion amplification coefficient (CPAC) and the research on system's stability, so learners don't know clearly how this parameter value is obtained. In this paper, firstly, the system stability which was the precondition for applying critical proportioning method was proved by using Rouse stability criterion and Bode diagram. Secondly, the CPAC value was deduced in theory according to Rouse stability criterion. Finally, a detailed example was given based on MATLAB, it enables learners to have a thorough understanding of the proposed method both in theory and simulation.
\end{abstract}

Keywords-critical proportioning method; Rouse stability criterion; critical proportion amplification coefficient

\section{INTRODUCTION}

PID control is the widest approach used in industrial process control community. PID control acts as the basis for many advanced process control. Since the control performance varies with PID parameters, PID parameters tuning is the core work for PID control systems. PID parameters tuning methods can be classified into the following two types [1]: 1) Theoretical calculation tuning method, which is dependent on mathematical modeling, and decides controller parameters by theoretical calculation. However, this approach exhibits some disadvantages in terms of the complicated calculation, the significant dependence on the mathematical modeling, and the computation data which can't be directly used and need to be adjusted according to practical engineering. 2) Engineering tuning method, which relies on engineering experiences and doesn't require the prior knowledge of the mathematical modeling of the control process, therefore can proceed on-site adjustment. This approach is simple and easy to be mastered, as a result, it can be commonly applied to practical engineering. Engineering tuning method mainly includes Ziegler-Nichols (Z-N) method, critical proportioning method and attenuation curve method.

In this paper, the critical proportioning method is used in PID parameters tuning. As a precondition for applying critical proportioning method, Rouse stability criterion and Bode diagram are used to present the system stability, then, the value of CPAC is deduced by Rouse stability criterion. From this work, the learners can know clearly how the value of CPAC is obtained, and have a thorough understanding of the critical proportioning method.

\section{THE EFFECT OF PID CONTROLLER PARAMETER ON CONTROL PERFORMANCE}

In a control system, PID algorithm can be expressed as

$$
u(t)=K_{\mathrm{P}}\left[e(t)+\frac{1}{T_{i}} \int_{0}^{t} e(t)+T_{\mathrm{d}} \frac{d e(t)}{d t}\right]
$$

The transfer function is

$$
G_{c}(s)=\frac{U(s)}{E(s)}=K_{\mathrm{p}}\left(1+\frac{1}{T_{i} s}+T_{\mathrm{d}} s\right)
$$

where $u(t)$ is control output, $e(t)$ denotes error signal, $K_{p}$ is proportion coefficient, $T_{i}$ is integration time constant and $T_{d}$ represents derivative time constant. The schematic of control system is shown in Figure I.

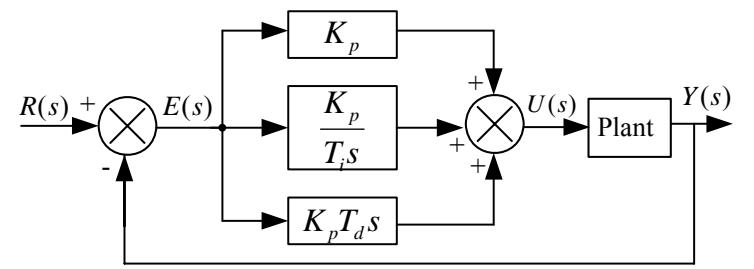

FIGURE I. THE SCHEMATIC OF PID CONTROL SYSTEM

1) Proportion coefficient $K_{P}$. Increasing $K_{P}$, the response of system will increase, it is beneficial to decrease the system static error and the effect of disturbance, but can't eliminate the static error ultimately. When $K_{\mathrm{P}}$ is large enough, overshoot and oscillation will occur, which lead to system unstable.

2) Integration time $T_{i}$. Decreasing $T_{i}$ will increase the system's response speed, decrease the overshoot, but it will increase the oscillation which degenerates the system stability.

3) Derivative time $T_{\mathrm{d}}$. Derivative control, which belongs to advanced control, is used to overcome larger transmission and capacity lags of the controlled plant, and to improve the system 
dynamic performances. The overshoot and transition time can be decreased if $T_{\mathrm{d}}$ is chosen appropriately.

From the above analysis, the three parameters of PID controller directly influence the control performance. In order to achieve satisfactory control performance, parameters $K_{p}$, $T_{i}$ and $T_{\mathrm{d}}$ should be calibrated in the most optimal manner.

\section{AdJUSTING PID CONTROLLER PARAMETERS USING CRITICAL PROPORTIONING METHOD}

The steps [2] of adjusting PID parameters by using critical proportioning method are summarized as the following.

1) Let the controller work in the pure $P$ control mode, namely set $T_{i}=\infty, T_{d}=0$, and then set the proportional band $\delta$ to a appropriate value, then system is operated by itself.

2) Changing the value of $\delta$ gradually from big to small until the system output presents persistent oscillation. This proportion band is called as the critical proportion band $\delta_{k}$, and the time interval between the two adjacent peaks is called as the critically oscillating period $T_{k}$, then compute and write down the values of $\delta_{k}$ and $T_{k}$.

3) According to the values of $\delta_{k}, T_{k}$ and the empirical formula shown in table I, we can compute the different values of parameters $K_{p}, T_{i}$ and $T_{d}$ corresponding to different controller types.

4) According to the operation order " $\mathrm{P} \rightarrow \mathrm{I} \rightarrow \mathrm{D}$ ", set controller parameters to the values achieved in step 3, then make the system work by itself. If the control performances are still not satisfied, further adjustments should be done according to the step 4 .

TABLE I. PID CONTROLLER PARAMETERS USING CPA

\begin{tabular}{|c|c|c|c|}
\hline $\begin{array}{c}\text { Controller } \\
\text { type }\end{array}$ & $\begin{array}{c}\text { Proportion } \\
\text { coefficient } \boldsymbol{K}_{\boldsymbol{p}}\end{array}$ & $\begin{array}{c}\text { Integration time } \\
\boldsymbol{T}_{\boldsymbol{i}}\end{array}$ & $\begin{array}{c}\text { Derivative time } \\
\boldsymbol{T}_{\boldsymbol{d}}\end{array}$ \\
\hline $\mathbf{P}$ & $\frac{1}{2 \delta_{k}}$ & $\infty$ & 0 \\
\hline PI & $\frac{1}{2.2 \delta_{k}}$ & $0.833 T_{k}$ & 0 \\
\hline PID & $\frac{1}{1.7 \delta_{k}}$ & $0.50 T_{k}$ & $0.125 T_{k}$ \\
\hline
\end{tabular}

\section{SIMULATION EXAMPLE}

Assume that there is a feedback system, whose open-loop transfer function is $G_{0}(s)=\frac{1}{s(s+1)(s+5)}$.In the following, the critical proportioning method is used to determine respectively the parameters of PID controller, and the unit step response curve of the closed-loop system is provided.

\section{A. Stability Analysis of The Closed-Loop System}

Since only stable system is controllable, the system stability analysis must be done before using the critical proportioning method to adjust the PID parameters.

1) Stability analysis of the closed-loop system using Rouse stability criterion [3]: In light of the simulation example, the transfer function of the open-loop system is given as

$$
G_{0}(s)=\frac{1}{s(s+1)(s+5)}
$$

Suppose that the transfer function of the open-loop system is $G(s)$, then

$$
G(s)=\frac{G_{0}(s)}{1+G_{0}(s)}=\frac{1}{s^{3}+6 s^{2}+5 s+1}
$$

and the system characteristic equation is obtained by $s^{3}+6 s^{2}+5 s+1=0$.

Now, let us list the Rouse table:

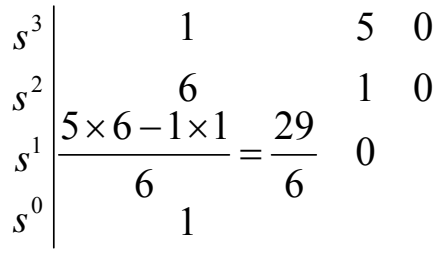

According to Rouse stability criterion, since all the coefficients of the system characteristic equation is positive and all the elements in the first column of the Rouse table are also positive, the closed-loop system is stable.

2) Stability analysis of the closed-loop system using Bode diagram: Since the transfer function of the open-loop system is $G_{0}(s)=\frac{1}{s(s+1)(s+5)}$, the frequency characteristic of the open-loop system is $G_{0}(j \omega)=\frac{1}{j \omega(1+j \omega)(5+j \omega)}$. On the other hand, since there is no open-loop pole on the right side, that is $P=0$ ( $P$ denotes the number of the pole of $G_{0}(s)$ in the right side $s$ domain plane), the open-loop system is stable. The system Bode diagram is presented in the following Figure II.

Analyzing the Bode diagram, it can conclude that in the frequency band of $M(\omega)>0, N_{-}^{\prime}$ and $N_{+}^{\prime}$ are equal to $\operatorname{zero}\left(N_{+}^{\prime}\right.$ is the number of the positive traversing, $N_{-}^{\prime}$ is the 
number of the negative traversing), therefore, $\frac{P}{2}=N_{+}^{\prime}-N_{-}^{\prime}$, and the closed-loop system is stable.

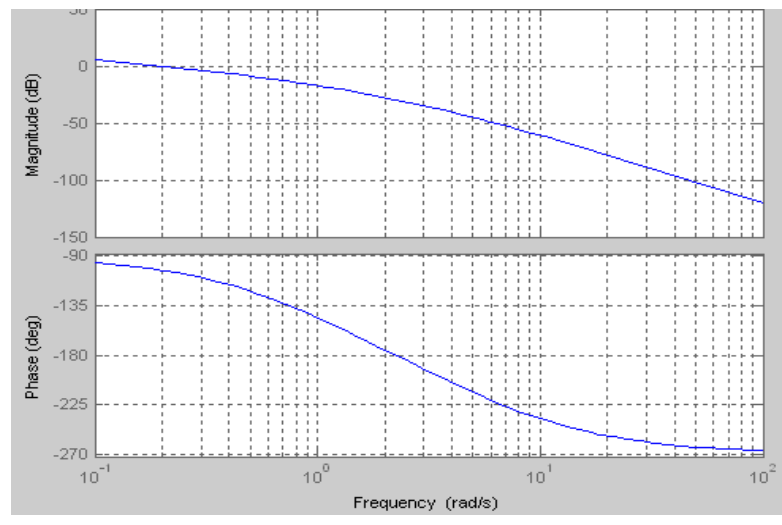

FIGURE II. BODE DIAGRAM

\section{B. System Simulation} below.

The model diagram of system simulation [4] is shown

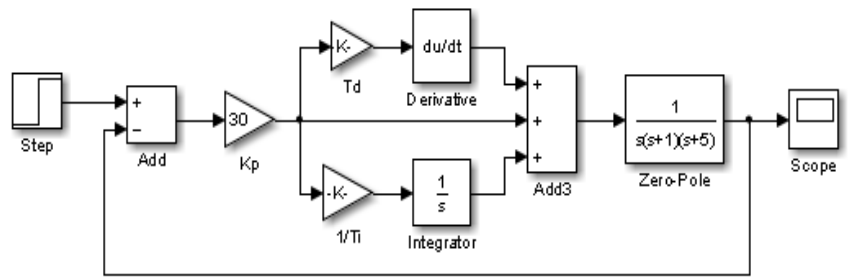

FIGURE III. SIMULINK MODEL DIAGRAM

a) Set $T_{i}=\infty, T_{d}=0$, and then set $\delta$ to a appropriate value, then system is operated by itself.

b) Adjusting appropriately the proportion coefficient $K_{\mathrm{P}}$ ( $K_{\mathrm{P}}=\frac{1}{\delta}$ ), until the system output presents persistent oscillation, computing and writing down the values of $\delta_{k}$ and $T_{k}$. Now, the major problem is what value of $K_{\mathrm{P}}$ should be set in theory when the system output presents persistent oscillation? We can use the Rouse stability criterion to decide the value of $K_{\mathrm{P}}$. The method is detailed as: regarding $\mathrm{P}$ controller and the controlled plant as the generalized controlled plant.

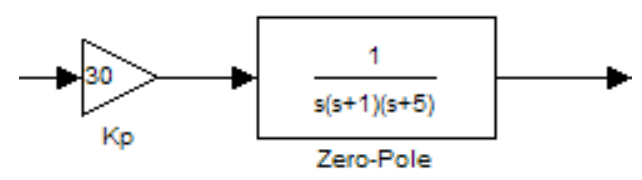

Whose generalized open-loop transfer function is $G_{0}^{\prime}(s)=\frac{K_{p}}{s(s+1)(s+5)}$, then the closed-loop transfer function of the generalized controlled plant is given by $G^{\prime}(s)=\frac{G_{0}^{\prime}(s)}{1+G_{0}^{\prime}(s)}=\frac{K_{p}}{s^{3}+6 s^{2}+5 s+K_{p}}$, and therefore the closed-loop system characteristic equation is $s^{3}+6 s^{2}+5 s+K_{p}=0$.

According to Rouse stability criterion, the necessary and sufficient conditions of a third-order closed-loop robust system are satisfied by

$$
\left\{\begin{array}{c}
K_{p}>0 \\
a_{1} a_{2}-a_{3} a_{0}>0
\end{array}\right.
$$

After substituting data ,the expression is

$$
5 \times 6-1 \times K_{p}>0
$$

The numerical-value of $K_{p}$ is $0<K_{p}<30$, hence $K_{p}^{\prime}=30$ is the CPAC. In turn, it indicates that when $K_{\mathrm{P}}$ is adjusted to 30 , the system output will present persistent oscillation, so the value of CPAC is determined in such a way.

To carry out computer simulation, we set $K_{\mathrm{P}}=30, \frac{1}{T_{i}}=0, T_{d}=0$, the simulation results are plotted in the following figure. Seen from the figure, the value of the critically oscillating period $T_{\mathrm{k}}$ is 2.81 .

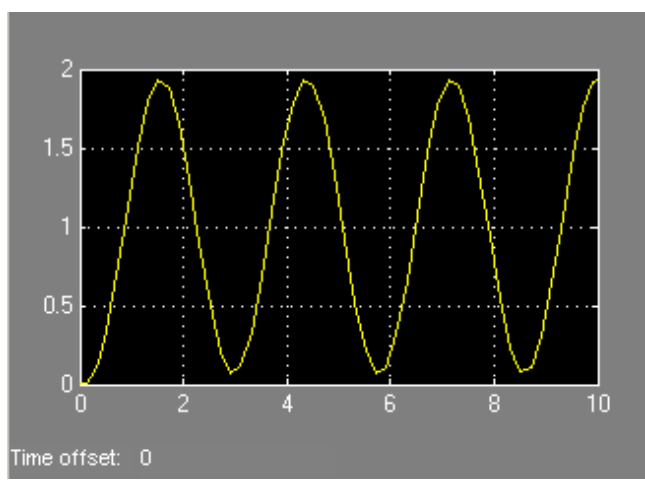

FIGURE IV. THE UNIT STEP RESPONSE CURVE OF THE SYSTEM UNDER PID CONTROLLER

c) According to the values of $\delta_{\mathrm{k}}, T_{\mathrm{k}}$ and the empirical formula in table I, we compute the different values of parameters $K_{p}, T_{i}$ and $T_{d}$ corresponding to different controller types.

For example, when PID controller is used, 


$$
\begin{gathered}
K_{p}=\frac{1}{1.7 \delta_{k}}=\frac{1}{1.7 \times \frac{1}{30}} \approx 17.647 \\
T_{i}=0.50 T_{k}=0.50 \times 2.81 \approx 1.405 \\
T_{d}=0.125 T_{k}=0.125 \times 2.81 \approx 0.351
\end{gathered}
$$

The unit step response curve of the system is shown in the following figure when the PID controller is applied.

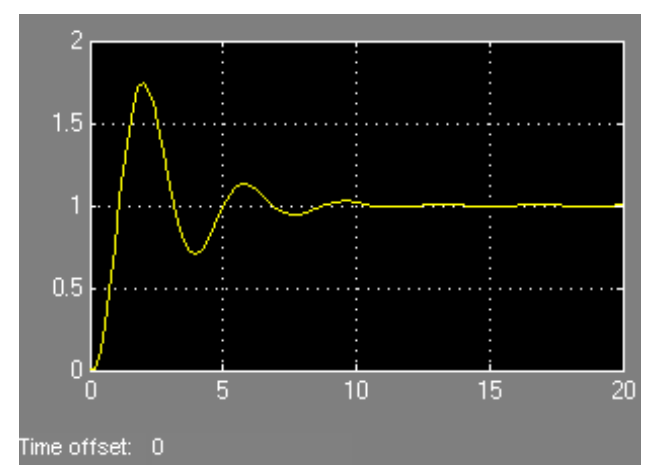

FIGURE V. THE UNIT STEP RESPONSE CURVE OF THE SYSTEM UNDER PID CONTROLLER

Since engineering tuning PID parameter method relies on empirical formula, it is unapplicable to any case, and therefore the PID parameters calibrated by engineering tuning method are not necessarily perfect. Meanwhile, if the PID parameters are not ideal, some adjustments should be made according to the practical process in order to obtain the satisfactory control performance.

\section{CONCLUSIONS}

System stability analysis has been discussed and judged by using Rouse stability criterion and Bode diagram. Then, the value of CPAC is deduced by Rouse stability criterion in theory, which saves a lot of regulation time in the process of PID parameters tuning. The simulation example is provided to give a comprehensive analysis and detailed steps of adjusting PID controller parameters by using critical proportioning method. Now, the learners can know clearly how the value of CPAC is obtained, and have a comprehensive, clear understanding of the critical proportioning method. Simulation results illustrate the effectiveness of the proposed method.

\section{REFERENCES}

[1] Hongcheng Sun etc. Process control engineering [M]. Beijing: Higher education press, 2006.

[2] Henglin Wang,Yangkuan Guo.MATLAB/Simulink and process control system simulation [M]. Beijing: Electronic industry press, 2012.

[3] Guoshen Gao etc.Principle of automatic control (2 rd) [M]. Guangzhou: South China university of technology press, 2005.
[4] Yueguang Sun etc."Simulation study on setting of PID controller parameters according to critical proportioning method," Modern Electronics Technique, [J]. China, vol.35 No.8, pp. 192-194, Apr. 2012. 